\title{
Metastable Congested States in Multisegment Traffic Cellular Automaton
}

\author{
Yutaka Nishimura $^{1}$, Taksu Cheon ${ }^{1}$ and Petr Šeba ${ }^{2}$ \\ ${ }^{1}$ Laboratory of Physics, Kochi University of Technology, Tosa Yamada, Kochi 782-8502, Japan \\ 2 Department of Physics, University of Hradec Kralove, Hradec Kralove CZ50003, Czech Republic
}

(Dated: November 8, 2005)

\begin{abstract}
We investigate a simple multisegment cellular automaton model of traffic flow. With the introduction of segment-dependent acceleration probability, metastable congested states in the intermediate density region emerge, and the initial-state dependence of the flow is observed. The essential feature of three-phased structure empirically found in real-world traffic flow is reproduced without elaborate assumptions.
\end{abstract}

PACS numbers: 02.70.+d, 05.70.Ln, 64.60.Cn

Keywords: cellular automaton, traffic flow, fluctuation

\section{INTRODUCTION}

The cellular automaton model has established itself as a standard in the analysis of traffic flow with its simplicity, affinity to computer simulations, and also with its extendibility to handle the realistic traffic [1, 2]. A particularly appealing aspect is its capability to capture the essential characteristics of the traffic flow with very few system parameters, which is a surprising economy of assumptions. With further assumptions for the adoption to the specific cases, the model is shown to be capable of reproducing down to the fine details of traffic flow in various settings, and it has been used for the analysis of real-life traffic with great success in past decade [3].

Lost in this process of elaboration, however, is the elegance of generality which the original standard model possesses. There is an obvious generic feature of the traffic flow that is beyond the reach of original model, but appears to await the explanation with a simple model. That is the three phase structure. In a typical traffic, the low and high density regions show the free motion and traffic jam. In the fundamental diagram of traffic, that plots the flux against the density, they are respectively represented by the increasing and decreasing lines, both of which are reproduced well with the standard model. In the intermediate density, however, empirical data indicate the existence of the third phase, in which there is no clear single line in the fundamental diagram, but the points are scattered in broad region, indicating the existence of the fluctuation in the traffic flow.

In this article, we intend to show that this third phase appears in a very simple extension of the standard model that includes segments which has different system parameters. These segments are introduced to represent the natural bottlenecks - narrow road segments, hilly segments, a segment under construction, etc. - that are ubiquitous in real-life traffic. We base our discussion primarily on numerical simulations and supplement them with simple intuitive physical arguments.

It has been recognized for some time that the fluctuation in the traffic flow is closely related to the the appearance of the metastable states [4, [5, 6]. We find that the emergence of the third phase is also a direct re- sult of the metastable states that appear with a minimal assumption in our model.

\section{TRAFFIC MODEL WITH PROBABILISTIC ACCELERATION IN MULTI-SEGMENTED ROAD}

We first lay out our model. A road is divided into a one-dimensional array of $L$ cells on which $M$ cars are placed. The location (i.e. cell number) and the velocity of the cars are represented by integers $x_{i}$ and $v_{i}$ with the car index $i=1,2, \ldots, M$. Cars are indexed in ascending order to the direction of the motion without overlap; $x_{1}<x_{2}<\ldots<x_{M}$. The road is considered to be circular, so that the $(L+1)$-th cell is identified with the fist cell. The road is subdivided into $S$ segments $\mathcal{S}_{1}=\left\{x \mid 0<x \leq L_{1}\right\}, \mathcal{S}_{2}=\left\{x \mid L_{1}<x \leq L_{1}+L_{2}\right\}, \ldots$, $\mathcal{S}_{S}=\left\{x \mid L_{1}+\ldots+L_{S-1}<x \leq L\right\}$, with the constraint on the sub-length $L_{1}+\ldots+L_{S}=L$. Each segment, $s=1, \ldots, S$, is assigned its own maximum velocity $U_{s}$ and non-acceleration probability $R_{s}$. The time evolution of the system in discrete time steps $t \rightarrow t+1$ is described by the following three consecutive updating for $v_{i}$ and $x_{i}$ $\in S_{s}$

1) Probabilistic acceleration : $v_{i} \longrightarrow \min \left(v_{i}+1, U_{s}\right)$ with probability $\left(1-R_{s}\right)$

2) Breaking to avoid collision :

$v_{i} \longrightarrow \min \left(v_{i}, x_{i+1}-x_{i}-1\right)$.

3) Advancement :

$x_{i} \longrightarrow x_{i}+v_{i}$.

The updating is done in parallel for all cars $i=1,2, \ldots M$ : Namely, the current position of the preceding car is used to calculate the distance $x_{i+1}-x_{i}-1$.

The model can be thought of as a variant of the standard Nagel-Schreckenberg (NS) model in that it follows the acceleration and deceleration step-by-step. In fact, if we change the ordering of "breaking" and "random deceleration" in the standard NS model, we obtain a model where cars accelerate, and then randomly decelerate. The model then becomes almost identical to ours, 
apart from the treatment of the cars running at the maximum velocities. This inverting of the order effectively prohibits the cars running in the jam from random deceleration, thus models the "heightened alertness" of drivers who have the preceding cars in their close sight. Our model is also related to the Fukui-Ishibashi (FI) model in that both employ probabilistic acceleration, which is in contrast to the NS model that treats the process by splitting it into acceleration and probabilistic deceleration.

Using the second step of the rule (1), we may define a jam as a block of cars in which the distance of neighboring cars is below the maximum velocity, A crucial feature of our model is the existence of the metastable block of jam made up of equi-spaced cars. This is a direct result of our model in which no further random deceleration occurs for cars inside the jam. The only limiting factor of the growth of this block is the percolation at the head of the block whose rate is proportional to $\left(1-R_{s}\right)$. This feature captures oft observed self-forming bottleneck of slow-moving cars in single-lane roads in real-life traffic. This could be regarded as a main justification for devising yet another variant of traffic cellular automaton model.

\section{NUMERICAL RESULTS}

We perform numerical simulations on the model presented above with one segment, two-segments, and six segments cases, the last one being the model of generic realistic traffic.

The macroscopic characteristics of traffic systems are best summarized with so-called fundamental diagram, which is the traffic flux $F$ plotted as the function of traffic density $\rho$, each of which are defined as

$$
\begin{aligned}
\rho & =\frac{M}{L} \\
F & =\rho \frac{1}{M}\left\langle\sum_{i=1}^{M} v_{i}\right\rangle_{t}=\frac{1}{L}\left\langle\sum_{i=1}^{M} v_{i}\right\rangle_{t} .
\end{aligned}
$$

Here, \langle\rangle$_{t}$ stands for the average over many time steps $t$.

We first show the results of the single-segment traffic in FIG. 1. The size of the road is set to be $L=200$ and the maximum velocity is chosen to be $U=8$. Fundamental diagram with various non-acceleration rates $R$ are shown. Each point in the graph represents the result of 20,000 iterations starting from a single random initial configuration. In the diagram, we observe three distinct regimes of traffic flow. In between the free traffic regime at low density and jamming regime at high density, there is a distinct third regime at intermediate density. Here, the flux displays the sensitive dependence on the initial condition, which is shown in the graph as jittery structures.

It is certainly possible to consider the ensemble of initial conditions among which the results could be averaged

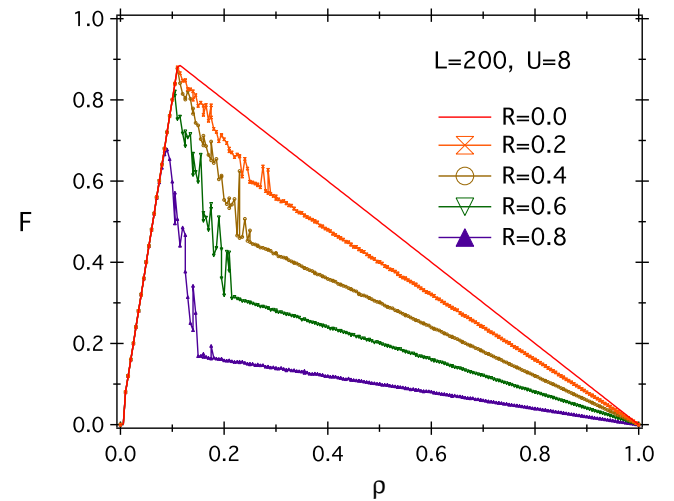

FIG. 1: The fundamental diagrams of our model. The nonacceleration rate $R$ is varied from $R=0$ to 0.8 as indicated in the graph. The maximum velocity is fixed at $U=8$ for all cases. The size of the road is set to be $L=200$.

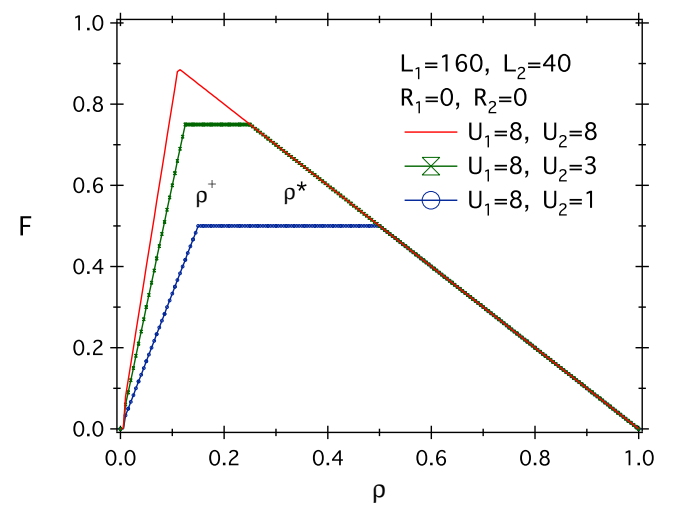

FIG. 2: The fundamental diagrams of two segment model with a common non-acceleration rates $R_{1}=R_{2}=0$ are shown. The maximum velocity for the first segment is fixed at $U_{1}=8$, and it is varied as $U_{2}=8,3$ and 1 for the second segment.

out to obtain smooth curves. However, we can also argue that jittery structure reflects a special feature of the model in which there can be metastable states that result in different flux for traffics with same density but different initial configurations. We stress that, at this stage, the existence of the intermediate region is a result of finite size effect. This can be easily proven by the explicit numerical calculation showing the shrinking of this region with larger $L$. At the limit $L \rightarrow \infty$, fundamental diagram with two phases with discontinuous $F(\rho)$ is obtained. However, this intermediate region develop into a distinct phase when there are more than two segments, $S \geq 2$ in a road.

That fact is clearly shown in FIG. 2, in which the fundamental diagram of two segment model with $L=200$ which is split into $L_{1}=160$ and $L_{2}=40$ is shown. Here, we set a common non-acceleration rate $R_{1}=R_{2}=0$ and different maximum velocities $U_{1}$ and $U_{2}$ for two segments. We assume the smaller maximum velocity for the second 
segment, $U_{1}>U_{2}$. By the inspection, we sense the existence of analytical solutions represented by the straight lines connecting $\rho=0, \rho=1$ both at $F=0$ and two critical densities $\rho^{\dagger}$ and $\rho^{\star}$ both at the plateau flux $F^{\star}$. At the critical density $\rho^{\dagger}$, the block of jam with $v_{i}=U_{2}$ and car spacing $x_{i+1}-x_{i}=U_{2}+1$ is formed in the second segment while the traffic is at full speed $v_{i}=U_{1}$ with car spacing $U_{1}+1$ in the first segment. At $\rho^{\star}$, however, the jam with $v_{i}=U_{2}$ fills the entire system. With elementary calculations, we obtain the critical densities in the forms

$$
\begin{aligned}
& \rho^{\dagger}=\frac{L_{1}}{L} \frac{1}{U_{1}+1}+\frac{L_{2}}{L} \frac{1}{U_{2}+1}, \\
& \rho^{\star}=\frac{1}{U_{2}+1} .
\end{aligned}
$$

We also obtain the plateau flux

$$
F^{\star}=\frac{U_{2}}{U_{2}+1} .
$$

At the plateau region $\rho^{\dagger} \leq \rho \leq \rho^{\star}$, the flow is limited by the block of jam with car spacing $U_{2}+1$ which now extends into the high maximum velocity region, in which drivers perceive the block as bottleneck congestion. The length of the block is determined by the requirement that "free traffic" segment has the density that corresponds to the same flux with the block, (6).

Note that the difference between two critical densities $\rho^{\dagger}$ and $\rho^{\star}$ persists at the continuum limit $L_{s} \rightarrow \infty$ if the ratio $L_{1} / L_{2}$ is kept constant. This clearly shows that the appearance of the intermediate plateau region is not the artifact of the discretization nor the finiteness of the model. Thus this third region can be legitimately regarded as the third phase. The three phase structure thus obtained is reminiscent to that obtained with the stop-and-go dynamics 8]. The metastable characteristics of the blocks is essential in obtaining this structure.

The situation becomes even more interesting when different non-acceleration rates are assigned to the different segments. In FIG. 3, the fundamental diagrams with a common $U_{s}$ and the different $R_{s}$ are shown. Actual values are chosen to be $U_{1}=U_{2}=8, R_{1}=0.1$ and $U_{2}=0.6$. Here, in between the free traffic phase at low density region $\rho<\rho^{\dagger}$ and jamming phase at high density region $\rho>\rho^{\star}$, we clearly observe an intermediate phase where there is wild oscillations in $F(\rho)$. Different outcome is obtained from different initial configuration. A most striking feature, however, is the fact that, in this intermediate phase, the values of the flux $F$ is discretized at the fractions

$$
F=\frac{V}{V+1}, \quad V=1,2, \ldots, U_{1} .
$$

The cause of this discretization and fluctuation is again the appearance of metastable blocks composed of equispaced cars moving in common velocity $V$. The road segment with high- $R_{s}$ hinders the percolation of the blocks,

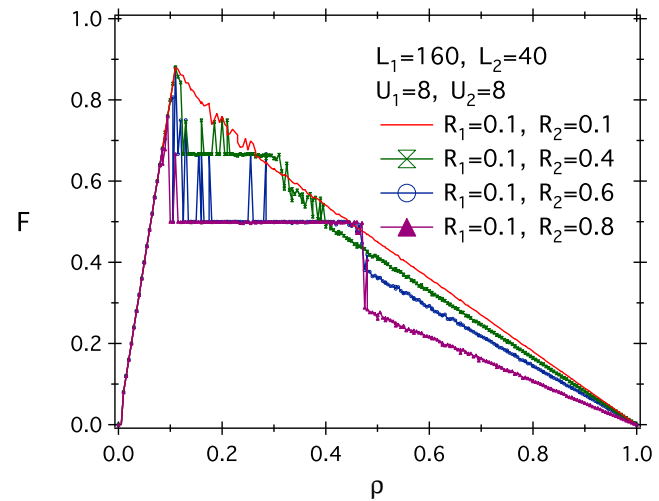

FIG. 3: The fundamental diagrams of two segment model with a common maximum velocity $U_{1}=U_{2}=8$ are shown. The non-acceleration rates for the first segment is fixed at $R_{1}=0.1$ and that of second segment is varied as $R_{2}=0.4$, 0.6 and 0.8 . Line connecting the data points are drawn solely to indicate the fluctuation between discretized flux: There are no points in-between the value specified by (7) in the intermediate density region.

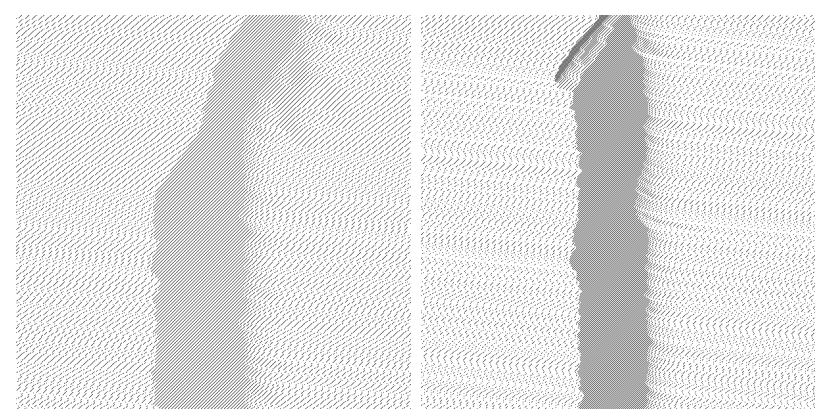

FIG. 4: The flow patterns of two segment model at $\rho=0.3$. The system parameters are $U_{1}=U_{2}=8, R_{1}=0.1$ and $R_{2}=$ 0.5. Different initial conditions result in different metastable states: The graph on the left hand side shows the block of jam with $v_{i}=4$, while the right hand side shows the one with $v_{i}=2$.

and helps the development of its aggregation. Thus, in effect, the high $R_{s}$ segment works very similar to the low- $U_{s}$ segment in previous example. A crucial difference, however, is that the block velocity $V$ is not preset. Metastable blocks with different $V$ can appear and dominate depending on the initial condition, which results in non-unique value for $F$ at each $\rho$.

Our claim of the appearance of plural metastable bottleneck blocks are corroborated by the flow pattern shown in FIG. 4, which is obtained from the same simulation as in FIG. 3. FIG. 4(a) and FIG. 4(b) are the results of same parameters but different initial conditions. clearly, metastable congestion blocks with different internal car spacing (thus different block velocities) are becoming the limiting factors of the total flow.

In order to further clarify the initial-condition dependence, we show the results of two different types of defi- 


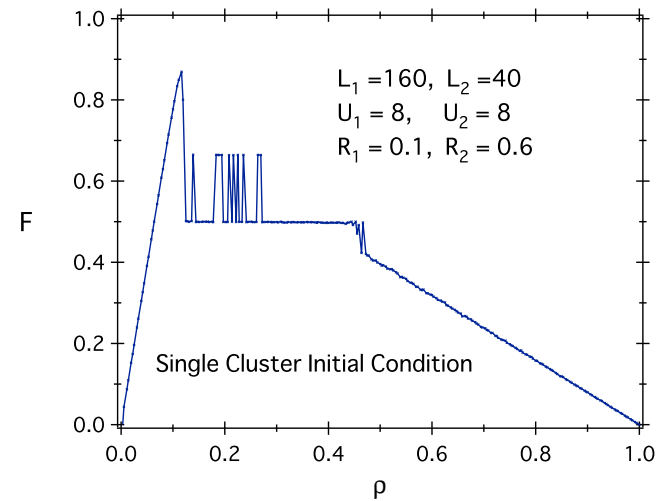

FIG. 5: The fundamental diagram of two segment model with a common maximum velocity $U_{1}=U_{2}=8$ and different nonacceleration rates $R_{1}=0.1, R_{2}=0.6$. The initial condition is taken to be a single cluster.

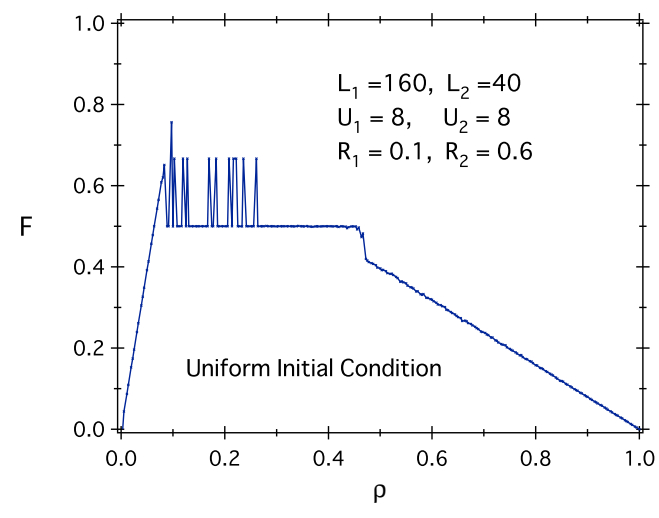

FIG. 6: Same as FIG. 5 except that the initial condition is taken to be uniformly distributed, equally spaced cars.

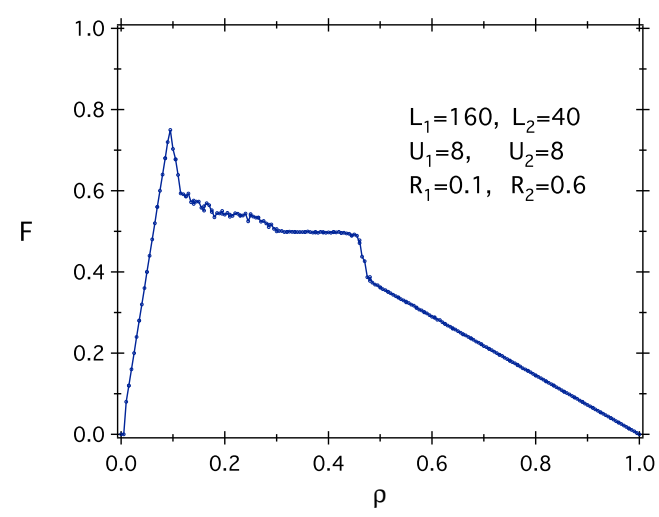

FIG. 7: The fundamental diagram of two segment model with a common maximum velocity $U_{1}=U_{2}=8$ and different non-acceleration rates $R_{1}=0.1, R_{2}=0.6$. The flux at each density is obtained by averaging over 200 different initial states.

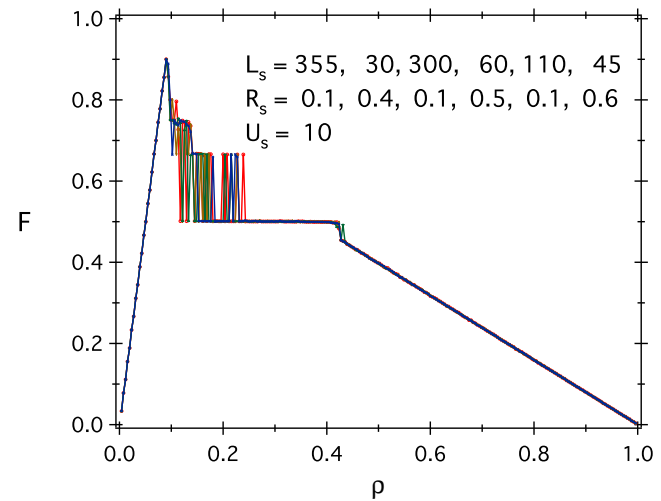

FIG. 8: The fundamental diagrams of "generic" six segment model with a common maximum velocity $U_{1}=\ldots=U_{6}=8$ are shown. The non-acceleration rates for the odd segments is fixed at $R_{1}=R_{3}=R_{5}=0.1$, and that of even segment is varied as $R_{2}=0.4, R_{4}=0.5$, and $R_{6}=0.6$. The lengths of the segments are $L_{1}=355, L_{2}=30, L_{3}=300, L_{4}=60$, $L_{5}=110$ and $L_{6}=45$. Here, results from a single random initial configuration is shown for each value of the density, as in FIG. 3.

nite initial states: In FIG. 5, the fundamental diagram of traffic flow calculated from single cluster initial states is shown. In FIG. 6, the initial condition is now a uniform distribution, meaning equally spaced cars spread over the whole road. In both cases, a common maximum velocities $U_{1}=U_{2}=8$ and different non-acceleration rates $R_{1}=0.1$ and $R_{2}=0.6$ are used. Even with these welldefined non-random initial conditions, they both show the fluctuating flux as the functions of density. These diagrams unambiguously show that the discretization is the results of random appearance of different metastable states. In FIG. 7, the result of average over different initial condition is shown. The system parameters are the same as those in FIG. 5 and FIG. 6. The flux now comes in between the discretized value found in the case of single initial condition. This is what we should expect when the fundamental diagram is drawn from the accumulation of actual data from real-life traffic.

We now move on to show that the existence of a phase with fluctuating discretized flux is not a specific result of the two-segment model, but a feature generic to multisegment traffic in our model. In FIG. 8, we present the results of six segment traffic, which is brought up as a toy model of realistic single-lane "county-road" traffic. Road length is set to be $L=900$. The road is now made up of the "normal" segments $s=1,3,5$ with $R_{1}=R_{3}=R_{5}=0.1$, and the bottleneck segments $s=2,4,6$, each having $R_{2}=0.4, R_{4}=0.5$ and $R_{6}=0.6$. The length of each segments are set to be $L_{1}=355$, $L_{2}=30, L_{3}=300, L_{4}=60, L_{5}=110$ and $L_{6}=45$. These arbitrary numbers are chosen to make this case a generic example. A common maximum velocity $U_{s}=10$ is used for all $s$. We find the result to be essentially indistinguishable to the case of two segments. In a sense, 
this is to be expected because if several jamming blocks are formed on a road with many segments, the one with smallest flux among them will eventually absorb the rest and becomes the limiting bottleneck, thus determine the total flow of the system. In this sense, analytical results of two segments with a common non-acceleration rate, (4)-(6), showing the existence of the "quantized" flux is the basis of all subsequent results showing the metastable jamming blocks.

\section{SUMMARY AND PROSPECTS}

In real-life traffic, we often experience different mode of congestion on different days on same roads with similar number of cars. In this article, we have shown that this puzzling feature is captured with a very simple cellular automaton model. Our model has a property of self-aggregating block of jam. This jam functions as a dynamical bottleneck, and leads to the existence of the third phase at intermediate density where visually striking non-unique flux is observed for a given density. It is our view, that among the multiple possible variants of original NS models, ones with such property are definitely worth further attention.

On the other hand, we want to make clear that the introduction of the alternative new rule (1) is not the prerequisite for the existence of the third phase. In fact, it can be shown through the numerical simulations that the three phase structure is already present in multisegment model with standard NS rules. With our modified rule that allows the formation of metastable blocks of jam, however, the new phase obtains distinct characteristics of discretized flux.
The existence of metastable states has been already observed in several traffic flow models $4,5,6]$ that effectively amount to introduce some extra rules to the standard Nagel-Schreckenberg model. In particular, early indication of discretized flux can be observed already in [6]. Our model is arguably the simplest of such extensions since it actually reduces the number of rules. More important point than the smaller number of rules is that, with our model, we are able to show a clear connection between the metastable states and the newly found intermediate phase featuring the discretized flux. In light of our results, it is suspected that the existence of the metastable states observed in elaborate realistic traffic models do not come from detailed model-specific assumptions but have a simple dynamics behind it which is captured in our minimal model.

The formation of metastable clusters is recently observed in a cellular automaton model of ants' trail [9]. It would be beneficial to compare our results with those of this work to gain a better insight into the dynamics of block of jam.

It is worth mentioning that the state of the system in the newly identified third regime at intermediate density shares many common features with so-called self-organized criticality [10]. It is as though the system is at critical point for extended range of parameter values with various competing metastable states formed spontaneously without fine-tuning of the system. It should be interesting to check, for example, the existence of $1 / \mathrm{f}$ type fluctuation on some measure, a hall mark of self-organized criticality, to further clarify the connection.
[1] K. Nagel and M. Schreckenberg, J. de Phys. I I2 (1992) 2221.

[2] M. Schreckenberg, A. Schadschneider, K. Nagel and N. Ito, Phys. Rev. E51 (1995) 2939.

[3] K. Nagel and C.L. Barrett, Int. J. Mod. Phys. C8 (1997) 505.

[4] R. Barlovic, L. Santen, A. Schadschneider, M. Schreckenberg, Eur. Phys. J. B5 (1998) 793.

[5] C. Appert and L. Santen, Phys. Rev. Lett. 86 (2001) 2498.

[6] K. Nishinari, M. Fukui and A. Schadschneider, J. Phys.
A37 (2004) 3101.

[7] M. Fukui and Y. Ishibashi, J. Phys. Soc. Jpn. 65 (1996) 1868.

[8] Y. Ishibashi and M. Fukui, J. Phys. Soc. Jpn. 70 (2001) 1237.

[9] K. Nishinari, D. Chowdhuri and A. Schadenschneider, Phys. Rev. E67 (2003) 036120.

[10] P. Bak, C. Tang and K. Wiesenfeld, Phys. Rev. Lett. 59 (1987) 381. 